\title{
Article \\ Zero-Hopf Bifurcation in a Generalized Genesio Differential Equation
}

\author{
Zouhair Diab $^{1}$, Juan L. G. Guirao ${ }^{2, *(\mathbb{C})}$ and Juan A. Vera ${ }^{3}$ \\ 1 Zouhair Diab Department of Mathematics and Computer Science, Larbi Tebessi University, Tebessa 12002, \\ Algeria; zouhair.diab@univ-tebessa.dz \\ 2 Departamento de Matemáca Aplicada y Estadística, Universidad Politécnica de Cartagena, 30202 Cartagena, \\ Región de Murcia, Spain \\ 3 Centro Universitario de la Defensa, Academia General del Aire, Universidad Politécnica de Cartagena, \\ 30720 Santiago de la Ribera, Región de Murcia, Spain; juanantonio.vera@upct.es \\ * Correspondence: juan.garcia@upct.es
}

check for updates

Citation: Diab, Z.; Guirao, J.L.G.; Vera, J.A. Zero-Hopf Bifurcation in a Generalized Genesio Differential Equation. Mathematics 2021, 9, 354. https://doi.org/10.3390/math9040354

Academic Editor: Maria Luz Gandarias and Rami Ahmad El-Nabulsi Received: 21 December 2020 Accepted: 2 February 2021 Published: 10 February 2021

Publisher's Note: MDPI stays neutral with regard to jurisdictional clai$\mathrm{ms}$ in published maps and institutional affiliations.

Copyright: (C) 2021 by the authors. Licensee MDPI, Basel, Switzerland. This article is an open access article distributed under the terms and conditions of the Creative Commons Attribution (CC BY) license (https:// creativecommons.org/licenses/by/ $4.0 /)$.

\begin{abstract}
The purpose of the present paper is to study the presence of bifurcations of zero-Hopf type at a generalized Genesio differential equation. More precisely, by transforming such differential equation in a first-order differential system in the three-dimensional space $\mathbb{R}^{3}$, we are able to prove the existence of a zero-Hopf bifurcation from which periodic trajectories appear close to the equilibrium point located at the origin when the parameters $a$ and $c$ are zero and $b$ is positive.
\end{abstract}

Keywords: Zero-Hopf bifurcation; periodic solutions; ordinary differential equation

MSC: 34C23; 34C25; 37G10

\section{Introduction and Statement of the Main Results}

In [1], the authors analyzed the presence of zero-Hopf bifurcations for the ordinary differential equation of order three

$$
\dddot{x}+a \ddot{x}+b \dot{x}+c x-x^{2}=0,
$$

which is commonly known as the Genesio equation.

In this work, our aim is to study the existence of bifurcations of zero-Hopf type at the so-called generalized Genesio differential equation

$$
\dddot{x}+a \ddot{x}+b \dot{x}+c x+h(x)=0,
$$

where $h(x)=\sum_{k=1}^{n}(-1)^{k} x^{2 k}$, where $n$ is any natural number, and $a, b, c \in \mathbb{R}$. Note that the map $h(x)$ is defined in the maximum domain where the solutions of (2) are defined. Note that for $n=1$, we have the Genesio equation; for $n=2$, we have the simplest chaotic jerk equation and so on. We underline that the results stated in this paper can be mimetically reproduced for a smooth and even $h(x)$.

By defining of the variables $y=\dot{x}$ and $z=\dot{y}$, the differential Equation (2) can be written as the system of nonlinear differential equations

$$
\left\{\begin{array}{l}
\dot{x}=y, \\
\dot{y}=z, \\
\dot{z}=-c x-b y-a z-\sum_{k=1}^{n}(-1)^{k} x^{2 k}
\end{array}\right.
$$

Our endeavor is is to analyze the presence of zero-Hopf type equilibria and the existence of zero-Hopf bifurcations in the system (3). 
Definition 1. An zero-Hopf type equilibrium for a three-dimensional autonomous differential system of first-order is an isolated equilibrium point of such a system for which its linear part has eigenvalues one equal to zero and two purely imaginary, see [2] for more details.

The main approach to prove the presence of a zero-Hopf bifurcation is to transform the system object of our study into the normal form of a zero-Hopf bifurcation. However, the treatment that we have chosen for the system (3) is via the use of the averaging theory of dynamical systems. In Section 2, a summary of the main results which play a key role in our study are stated. The averaging method has already been used to study-Hopf and zero-Hopf bifurcations for other differential systems, see for instance [3-6]. Our main results are as follows:

Proposition 1. The differential system (3) has a unique equilibrium of zero-Hopf type located at the origin when $a=c=0$ and $b>0$.

Theorem 1. Let us consider for the system (3), the following distribution of parameters $a=\varepsilon \alpha$, $b=\omega^{2}+\varepsilon \beta$ and $c=\varepsilon \gamma$, being $\omega>0$ and $\varepsilon$ a sufficiently small parameter. Then, (3) has a zero-Hopf type bifurcation at the origin of coordinates in its equilibrium point for $\varepsilon=0$. In the case $\gamma^{2}-\alpha^{2} \omega^{4}>0$, (3) presents a unique periodic trajectory bifurcating from the origin. When $\gamma=\alpha \omega^{2}$, (3) has at most 6 periodic orbits bifurcating from the origin.

\section{Preliminaries}

\subsection{Results from Averaging Theory}

Here, we present the main results on the second-order averaging theory of dynamical systems that will play a key role in the proof of our main results Theorem 1. For more information on this interesting theory and its application, see for instance [7] or [8] and references therein. For a proof of Theorem 2 that we are going to state, see Theorem 3.5.1 of Sanders and Verhulst [2], or [9] for a formulation in modern terminology.

Consider the differential system

$$
\dot{x}=\varepsilon F_{1}(t, x)+\varepsilon^{2} F_{2}(t, x)+\varepsilon^{3} Q(t, x, \varepsilon), \quad x(0)=x_{0},
$$

where $F_{1}, F_{2}: \mathbb{R} \times \Omega \rightarrow \mathbb{R}^{n}$ and $Q:[0, \infty] \times \Omega \times\left(0, \varepsilon_{0}\right] \rightarrow \mathbb{R}^{n}$ where $\Omega$ is a subset of $\mathbb{R}^{n}$ open and $F_{1}, F_{2}$ and $Q$ are maps periodic of period $T$ in the first variable. We set

$$
\begin{aligned}
& F_{10}(x)=\frac{1}{T} \int_{0}^{T} F_{1}(t, x) d t, \\
& F_{20}(x)=\frac{1}{T} \int_{0}^{T}\left[D_{x} F_{1}(t, x) \cdot y_{1}(t, x)+F_{2}(t, x)\right] d t,
\end{aligned}
$$

where

$$
y_{1}(t, x)=\int_{0}^{t} F_{1}(s, x) d s,
$$

Theorem 2. In the previous conditions, assume that

(i) $F_{1}, F_{2}, \partial F_{1} / \partial x$ are locally Lipschitz in $x$ and $Q$ is twice differentiable with respect to $\varepsilon$.

(ii) Let $V \subset \Omega$ be a bounded and open set, for each $\varepsilon \in\left(-\varepsilon_{f}, \varepsilon_{f}\right) \backslash\{0\}$, there exists $a_{\varepsilon} \in V$ such that $F_{10}\left(a_{\varepsilon}\right)+\varepsilon F_{20}\left(a_{\varepsilon}\right)=0$ and $d_{B}\left(F_{10}+\varepsilon F_{20}, V, a_{\varepsilon}\right) \neq 0$.

Thus, for sufficiently small $\varepsilon>0$ there exists a T-periodic solution $\varphi(\cdot, \varepsilon)$ holding $\varphi(0, \varepsilon)=a_{\varepsilon}$.

Remark 1. When we write $d_{B}\left(F_{10}+\varepsilon F_{20}, V, a_{\varepsilon}\right) \neq 0$ means that the value of the Brouwer degree of the function $F_{10}: V \rightarrow \mathbb{R}^{n}$ at the fixed point $a_{\varepsilon}$ is not zero. A sufficient condition for having the previous property is that the Jacobian of the function $F_{10}+\varepsilon F_{20}$ at $a_{\varepsilon}$ be non-zero. When $F_{10} \neq 0$, then the zeros of $F_{10}+\varepsilon F_{20}$ are equivalent to the zeros of $F_{10}$ for sufficiently small $\varepsilon$. In this case, we 
can apply the so-called averaging theory of first-order dynamical systems. In the case $F_{10}=0$ but $F_{20} \neq 0$, the zeros of $F_{10}+\varepsilon F_{20}$ are the ones of $F_{20}$ for sufficiently small $\varepsilon$. In such cases, we must apply second-order averaging theory.

\section{Proof of Proposition 1 and Theorem 1}

Proof of Proposition 1. We are going to see that the linear part of the characteristic polynomial of system (3) at the equilibrium point $x_{c}=(c, 0,0)$ is $q(\lambda)=\lambda^{3}+a \lambda^{2}+b \lambda+c$. We shall find the values of the parameters for which $q$ has an eigenvalue equal to zero and a pair of purely imaginary roots, i.e., values for which $q$ is of the form $-\lambda\left(\lambda^{2}+b\right)$ with $b>0$.

To simplify, let $b=\omega^{2}$, with $\omega>0$. Thus, if we assume $q(\lambda)=-\lambda\left(\lambda^{2}+\omega^{2}\right)$, we obtain that $a=c=0$ and $b>0$. Therefore, when $a=c=0$ and $b>0$, there exists an equilibrium point at the origin of unique coordinates of zero-Hopf type. Moreover, if we consider $b=\omega^{2}$, for $\omega>0$, then the eigenvalues are equal to 0 and $\pm i \omega$, ending the proof of Proposition 1.

Proof of Theorem 1. We are going to use the ideas of the second-order averaging theory of dynamical systems that we have briefly exposed in Section 2.1 (see Theorem 2) for analyzing the existence of equilibrium points of zero-Hopf type at the origin. Recall that such zero-Hopf type equilibrium points bifurcate some periodic orbit by moving the parameters $a, b, c$ of system (3). In this order of ideas, let us consider $a=\varepsilon \alpha, b=\omega^{2}+\varepsilon \beta$, $c=\varepsilon \gamma$ with $\varepsilon>0$ being a sufficiently small parameter. Then, the system (3) becomes

$$
\left\{\begin{array}{l}
\dot{x}=y, \\
\dot{y}=z, \\
\dot{z}=-\varepsilon \gamma x-\left(\omega^{2}+\varepsilon \beta\right) y-\varepsilon \alpha z-\sum_{k=1}^{n}(-1)^{k} x^{2 k}
\end{array}\right.
$$

The first step in order to write our differential system (6) in the normal form for applying the averaging theory is to write the linear part at the origin of system (6) when $\varepsilon=0$ as its real Jordan normal form, which is of the form

$$
\left(\begin{array}{ccc}
0 & -\omega & 0 \\
\omega & 0 & 0 \\
0 & 0 & 0
\end{array}\right) .
$$

Applying this linear change of variables $(x, y, z) \rightarrow(X, Y, Z)$ where

$$
x=\frac{Z-\omega X}{\omega^{2}}, y=Y, z=\omega X .
$$

In the new variables $(X, Y, Z)$, system (6) becomes 


$$
\begin{aligned}
\dot{X}= & \left(\gamma \omega^{11} X-\alpha \omega^{13} X-\gamma \omega^{10} Z-\gamma \omega^{12} \beta\right) \varepsilon / \omega^{13} \\
& +\left(\omega^{6} X^{6}-6 \omega^{5} X^{5} Z-\gamma \omega^{14}-20 \omega^{3} X^{3} Z^{3}\right. \\
& +15 \omega^{2} X^{2} Z^{4}+\omega^{10} X^{2}-2 \omega^{9} X Z+\omega^{8} Z^{2} \\
& -\omega^{8} X^{4}+4 \omega^{7} X^{3} Z-6 \omega^{6} X^{2} Z^{2} \\
& +4 \omega^{5} X Z^{3}-\omega^{4} Z^{4}-6 \omega X Z^{5}+15 \omega^{4} X^{4} Z^{2} \\
& \left.+Z^{6}-\sum_{k=4}^{n}(-1)^{k}\left(\frac{Z-\omega X}{\omega^{2}}\right)^{2 k} \omega^{12}\right) / \omega^{13}, \\
\dot{Y}= & \omega X, \\
\dot{Z}= & \left(\gamma \omega^{11} X-\alpha \omega^{13} X-\gamma \omega^{10} Z-\gamma \omega^{12} \beta\right) \varepsilon / \omega^{12} \\
& -\left(6 \omega^{5} X^{5} Z+15 \omega^{4} X^{4} Z^{2}-20 \omega^{3} X^{3} Z^{3}\right. \\
& +15 \omega^{2} X^{2} Z^{4}+\omega^{10} X^{2}-2 \omega^{9} X Z+\omega^{8} Z^{2} \\
& -\omega^{8} X^{4}+4 \omega^{7} X^{3} Z-6 \omega^{6} X^{2} Z^{2} \\
& +4 \omega^{5} X Z^{3}-\omega^{4} Z^{4}+\omega^{6} X^{6}-6 \omega X Z^{5} \\
& \left.+Z^{6}-\sum_{k=4}^{n}(-1)^{k}\left(\frac{Z-\omega X}{\omega^{2}}\right)^{2 k} \omega^{12}\right) / \omega^{12} .
\end{aligned}
$$

Doing a re-scale of the variables $(X, Y, Z)$ in the form $(X, Y, Z) \rightarrow(\varepsilon u, \varepsilon v, \varepsilon w)$, then system (8) is expressed as follows

$$
\begin{aligned}
\dot{u}= & -\omega v+\left(\gamma \omega^{11} u-\alpha \omega^{13} u+\omega^{10} u^{2}-\gamma \omega^{10} w-v \omega^{12} \beta-2 \omega^{9} u w+\omega^{8} w^{2}\right) \varepsilon / \omega^{13} \\
& +\left(-\omega^{8} u^{4}-\omega^{4} w^{4}+4 \omega^{7} u^{3} w-6 \omega^{6} u^{2} w^{2}+4 \omega^{5} u w^{3}\right) \varepsilon^{3} / \omega^{13} \\
& +\left(\omega^{6} u^{6}-6 \omega^{5} u^{5} w+15 \omega^{2} u^{2} w^{4}-6 \omega u w^{5}+15 \omega^{4} u^{4} w^{2}\right. \\
& \left.-20 \omega^{3} u^{3} w^{3}+w^{6}\right) \varepsilon^{5} / \omega^{13}-\frac{1}{\omega} \sum_{k=4}^{n}(-1)^{k}\left(\frac{(w-\omega u)}{\omega^{2}}\right)^{2 k} \varepsilon^{2 k-1}, \\
\dot{v}= & \omega u, \\
\dot{w}= & \left(\gamma \omega^{11} u-\alpha \omega^{13} u-\gamma \omega^{10} w-v \omega^{12} \beta+\omega^{10} u^{2}-2 \omega^{9} u w+\omega^{8} w^{2}\right) \varepsilon / \omega^{12} \\
& +\left(-\omega^{8} u^{4}-\omega^{4} w^{4}+4 \omega^{7} u^{3} w-6 \omega^{6} u^{2} w^{2}+4 \omega^{5} u w^{3}\right) \varepsilon^{3} / \omega^{12} \\
& +\left(\omega^{6} u^{6}-6 \omega^{5} u^{5} w+15 \omega^{2} u^{2} w^{4}-6 \omega u w^{5}+15 \omega^{4} u^{4} w^{2}\right. \\
& \left.-20 \omega^{3} u^{3} w^{3}+w^{6}\right) \varepsilon^{5} / \omega^{12}-\sum_{k=4}^{n}(-1)^{k}\left(\frac{(w-\omega u)}{\omega^{2}}\right)^{2 k} \varepsilon^{2 k-1} .
\end{aligned}
$$

The previous system can be written as follows 


$$
\begin{aligned}
\dot{u}= & -\omega v+\left(\gamma \omega^{11} u-\alpha \omega^{13} u+\omega^{10} u^{2}-\gamma \omega^{10} w\right. \\
& \left.-v \omega^{12} \beta-2 \omega^{9} u w+\omega^{8} w^{2}\right) \varepsilon / \omega^{13}+\left(-\omega^{8} u^{4}\right. \\
& \left.-\omega^{4} w^{4}+4 \omega^{7} u^{3} w-6 \omega^{6} u^{2} w^{2}+4 \omega^{5} u w^{3}\right) \varepsilon^{3} / \omega^{13} \\
& +\left(\omega^{6} u^{6}-6 \omega^{5} u^{5} w+15 \omega^{2} u^{2} w^{4}-6 \omega u w^{5}\right. \\
& \left.+15 \omega^{4} u^{4} w^{2}-20 \omega^{3} u^{3} w^{3}+w^{6}\right) \varepsilon^{5} / \omega^{13}+O\left(\varepsilon^{7}\right) \\
\dot{v}= & \omega u \\
\dot{w}= & \left(\gamma \omega^{11} u-\alpha \omega^{13} u-\gamma \omega^{10} w-v \omega^{12} \beta+\omega^{10} u^{2}\right. \\
& \left.-2 \omega^{9} u w+\omega^{8} w^{2}\right) \varepsilon / \omega^{12}+\left(-\omega^{8} u^{4}-\omega^{4} w^{4}\right. \\
& \left.+4 \omega^{7} u^{3} w-6 \omega^{6} u^{2} w^{2}+4 \omega^{5} u w^{3}\right) \varepsilon^{3} / \omega^{12} \\
& +\left(\omega^{6} u^{6}-6 \omega^{5} u^{5} w+15 \omega^{2} u^{2} w^{4}-6 \omega u w^{5}\right. \\
& \left.+15 \omega^{4} u^{4} w^{2}-20 \omega^{3} u^{3} w^{3}+w^{6}\right) \varepsilon^{5} / \omega^{12}+O\left(\varepsilon^{7}\right) .
\end{aligned}
$$

Now, we express the differential system (9) in cylindrical coordinates $(r, \theta, w)$ defined by $u=r \cos \theta$ and $v=r \sin \theta$, and we obtain

$$
\begin{aligned}
\dot{r}= & \cos (\theta)\left(\gamma \omega^{11} r \cos (\theta)-\alpha \omega^{13} r \cos (\theta)+\omega^{10} r^{2}(\cos (\theta))^{2}\right. \\
& \left.-\gamma \omega^{10} w-r \sin (\theta) \omega^{12} \beta-2 \omega^{9} r \cos (\theta) w+\omega^{8} w^{2}\right) \varepsilon / \omega^{13} \\
& +\cos (\theta)\left(-\omega^{8} r^{4}(\cos (\theta))^{4}-\omega^{4} w^{4}+4 \omega^{7} r^{3}(\cos (\theta))^{3} w\right. \\
& \left.-6 \omega^{6} r^{2}(\cos (\theta))^{2} w^{2}+4 \omega^{5} r \cos (\theta) w^{3}\right) \varepsilon^{3} / \omega^{13} \\
& +\cos (\theta)\left(\omega^{6} r^{6}(\cos (\theta))^{6}-6 \omega^{5} r^{5}(\cos (\theta))^{5} w\right. \\
& +15 \omega^{2} r^{2}(\cos (\theta))^{2} w^{4}-6 \omega r \cos (\theta) w^{5} \\
& +15 \omega^{4} r^{4}(\cos (\theta))^{4} w^{2}-20 \omega^{3} r^{3}(\cos (\theta))^{3} w^{3} \\
& \left.+w^{6}\right) \varepsilon^{5} / \omega^{13}+O\left(\varepsilon^{7}\right), \\
\dot{\theta}= & \omega-\left(-\sin (\theta) \omega^{13} \alpha r \cos (\theta)+\sin (\theta) \omega^{10} r^{2}(\cos (\theta))^{2}\right. \\
& -\sin (\theta) \gamma \omega^{10} w+\sin (\theta) \omega^{8} w^{2}+\omega^{12} r \beta(\cos (\theta))^{2} \\
& \left.-\omega^{12} r \beta-2 \sin (\theta) \omega^{9} r \cos (\theta) w+\sin (\theta) \omega^{11} r r \cos (\theta)\right) \varepsilon / r \omega^{13} \\
& -\left(4 \sin (\theta) \omega^{7} r^{3}(\cos (\theta))^{3} w-6 \sin (\theta) \omega^{6} r^{2}(\cos (\theta))^{2} w^{2}\right. \\
& +4 \sin (\theta) \omega^{5} r \cos (\theta) w^{3}-\sin (\theta) \omega^{4} w^{4} \\
& \left.-\sin (\theta) \omega^{8} r^{4}(\cos (\theta))^{4}\right) \varepsilon^{3} / r \omega^{13} \\
& -\left(\sin (\theta) \omega^{6} r^{6}(\cos (\theta))^{6}-6 \sin (\theta) \omega^{5} r^{5}(\cos (\theta))^{5} w\right. \\
& +15 \sin (\theta) \omega^{2} r^{2}(\cos (\theta))^{2} w^{4} \\
& +15 \sin (\theta) \omega^{4} r^{4}(\cos (\theta))^{4} w^{2}-20 \sin (\theta) \omega^{3} r^{3}(\cos (\theta))^{3} w^{3} \\
& \left.+\sin (\theta) w^{6}-6 \sin (\theta) \omega r \cos (\theta) w^{5}\right) \varepsilon^{5} / r \omega^{13}++O\left(\varepsilon^{7}\right), \\
= & \left(\gamma \omega^{11} r \cos (\theta)-\alpha \omega^{13} r \cos (\theta)+\omega^{10} r^{2}(\cos (\theta))^{2}-\gamma \omega^{10} w\right. \\
& \left.-r \sin (\theta) \omega^{12} \beta-2 \omega^{9} r \cos (\theta) w+\omega^{8} w^{2}\right) \varepsilon / \omega^{12} \\
& +\left(-\omega^{8} r^{4}(\cos (\theta))^{4}-\omega^{4} w^{4}+4 \omega^{7} r^{3}(\cos (\theta))^{3} w\right. \\
& \left.-6 \omega^{6} r^{2}(\cos (\theta))^{2} w^{2}+4 \omega^{5} r \cos (\theta) w^{3}\right) \varepsilon^{3} / \omega^{12} \\
& +\left(\omega^{6} r^{6}(\cos (\theta))^{6}-6 \omega^{5} r^{5}(\cos (\theta))^{5} w+15 \omega^{2} r^{2}(\cos (\theta))^{2} w^{4}\right. \\
& -6 \omega r \cos (\theta) w^{5}+15 \omega^{4} r^{4}(\cos (\theta))^{4} w^{2} \\
& \left.-20 \omega^{3} r^{3}(\cos (\theta))^{3} w^{3}+w^{6}\right) \varepsilon^{5} / \omega^{12}+O\left(\varepsilon^{7}\right) . \\
\dot{w} &
\end{aligned}
$$


In (10) we consider a new independent variable, and we obtain

$$
\begin{aligned}
& \frac{d r}{d \theta}=\varepsilon F_{11}(\theta, r, w)+\varepsilon F_{21}(\theta, r, w)+O\left(\varepsilon^{2}\right), \\
& \frac{d w}{d \theta}=\varepsilon F_{12}(\theta, r, w)+\varepsilon F_{22}(\theta, r, w)+O\left(\varepsilon^{2}\right),
\end{aligned}
$$

where

$$
\begin{aligned}
F_{11}(\theta, r, w)= & \cos (\theta)\left(\gamma \omega^{3} r \cos (\theta)-\alpha \omega^{5} r \cos (\theta)+\omega^{2} r^{2}(\cos (\theta))^{2}\right. \\
& \left.-\gamma \omega^{2} w-r \sin (\theta) \omega^{4} \beta-2 \omega r \cos (\theta) w+w^{2}\right) / \omega^{6}, \\
F_{12}(\theta, r, w)= & \gamma \omega^{3} r \cos (\theta)-\alpha \omega^{5} r \cos (\theta)+\omega^{2} r^{2}(\cos (\theta))^{2} \\
& \left.-\gamma \omega^{2} w-r \sin (\theta) \omega^{4} \beta-2 \omega r \cos (\theta) w+w^{2}\right) / \omega^{5},
\end{aligned}
$$

and

$$
\begin{aligned}
F_{21}(\theta, r, w)= & \sin (\theta) \cos (\theta)\left((\cos (\theta))^{4} \omega^{4} r^{4}-2 \cos (\theta) \omega^{5} \gamma^{2} r w\right. \\
& +(\cos (\theta))^{2} \omega^{6} \gamma^{2} r^{2}+2 \sin (\theta) \omega^{9} r^{2} \beta \cos (\theta) \alpha \\
& +6 \cos (\theta) \omega^{3} \gamma r w^{2}-2(\cos (\theta))^{2} \omega^{8} \gamma r^{2} \alpha \\
& -6(\cos (\theta))^{2} \omega^{4} r^{2} \gamma w+4(\cos (\theta))^{2} \omega^{6} \alpha r^{2} w \\
& -2 \cos (\theta) \omega^{5} \alpha r w^{2}-2(\cos (\theta))^{3} \omega^{7} \alpha r^{3} \\
& -4(\cos (\theta))^{3} \omega^{3} r^{3} w+w^{4}+\omega^{8} r^{2} \beta^{2} \\
& -4 \cos (\theta) \omega r w^{3}+\omega^{4} \gamma^{2} w^{2}+2 \cos (\theta) \omega^{7} \alpha r \gamma w \\
& -2 \sin (\theta) \omega^{4} w^{2} r \beta-2 \sin (\theta) \omega^{6} r^{3} \beta(\cos (\theta))^{2} \\
& +2(\cos (\theta))^{3} \omega^{5} \gamma r^{3}+(\cos (\theta))^{2} \omega^{10} \alpha^{2} r^{2} \\
& -(\cos (\theta))^{2} \omega^{8} r^{2} \beta^{2}+6(\cos (\theta))^{2} \omega^{2} r^{2} w^{2} \\
& -2 \omega^{2} w^{3} \gamma-2 \sin (\theta) \omega^{7} r^{2} \beta \cos (\theta) \gamma \\
& \left.+4 \sin (\theta) \omega^{5} r^{2} \beta \cos (\theta) w+2 \sin (\theta) \omega^{6} \gamma w r \beta\right) / \omega^{12} r \\
F_{22}(\theta, r, w)= & \sin (\theta) \cos (\theta)\left((\cos (\theta))^{4} \omega^{4} r^{4}-2 \cos (\theta) \omega^{5} \gamma^{2} r w\right. \\
& +(\cos (\theta))^{2} \omega^{6} \gamma^{2} r^{2}+2 \sin (\theta) \omega^{9} r^{2} \beta \cos (\theta) \alpha \\
& +6 \cos (\theta) \omega^{3} \gamma r w^{2}-2(\cos (\theta))^{2} \omega^{8} \gamma r^{2} \alpha \\
& -6(\cos (\theta))^{2} \omega^{4} r^{2} \gamma w+4(\cos (\theta))^{2} \omega^{6} \alpha r^{2} w \\
& -2 \cos (\theta) \omega^{5} \alpha r w^{2}-2(\cos (\theta))^{3} \omega^{7} \alpha r^{3} \\
& -4(\cos (\theta))^{3} \omega^{3} r^{3} w+w^{4}+\omega^{8} r^{2} \beta^{2} \\
& -4 \cos (\theta) \omega r w^{3}+\omega^{4} \gamma^{2} w^{2}+2 \cos (\theta) \omega^{7} \alpha r \gamma w \\
& -2 \sin (\theta) \omega^{4} w^{2} r \beta-2 \sin (\theta) \omega^{6} r^{3} \beta(\cos (\theta))^{2} \\
& +2(\cos (\theta))^{3} \omega^{5} \gamma r^{3}+(\cos (\theta))^{2} \omega^{10} \alpha^{2} r^{2} \\
& -(\cos (\theta))^{2} \omega^{8} r^{2} \beta^{2}+6(\cos (\theta))^{2} \omega^{2} r^{2} w^{2} \\
& -2 \omega^{2} w^{3} \gamma-2 \sin (\theta) \omega^{7} r^{2} \beta \cos (\theta) \gamma \\
& \left.+4 \sin (\theta) \omega^{5} r^{2} \beta \cos (\theta) w+2 \sin (\theta) \omega^{6} \gamma w r \beta\right) / \omega^{12} r
\end{aligned}
$$

With the notation introduced in Section 2.1, $t=\theta, T=2 \pi, x=(r, w)^{T}$ and

$$
\begin{gathered}
F_{1}(\theta, r, w)=\left(\begin{array}{l}
F_{11}(\theta, r, w) \\
F_{12}(\theta, r, w)
\end{array}\right) \quad \text { and } \quad F_{2}(\theta, r, w)=\left(\begin{array}{c}
F_{21}(\theta, r, w) \\
F_{22}(\theta, r, w)
\end{array}\right) . \\
F_{10}(r, w)=\left(\begin{array}{l}
F_{101}(r, w) \\
F_{102}(r, w)
\end{array}\right) \quad \text { and } \quad F_{20}(r, w)=\left(\begin{array}{l}
F_{201}(r, w) \\
F_{201}(r, w)
\end{array}\right) .
\end{gathered}
$$


It can easily be checked that (11) satisfies all the assumptions of Theorem 2. Computing the integrals (5), we have that

$$
\begin{aligned}
& F_{101}(r, w)=\frac{1}{2 \pi} \int_{0}^{2 \pi} F_{11}(\theta, r, w) d \theta=\frac{r\left(-2 w+\gamma \omega^{2}-\alpha \omega^{4}\right)}{2 \omega^{5}}, \\
& F_{102}(r, w)=\frac{1}{2 \pi} \int_{0}^{2 \pi} F_{12}(\theta, r, w) d \theta=-\frac{2 \gamma \omega^{2} w-\omega^{2} r^{2}-2 w^{2}}{2 \omega^{5}} .
\end{aligned}
$$

Note that $F_{101}(r, w)=F_{102}(r, w)=0$ has only one solution $(r, w)$ with $r^{*}>0$, more precisely

$$
r^{*}=\frac{\sqrt{-2 \omega^{4} \alpha^{2}+2 \gamma^{2}} \omega}{2}, w^{*}=\frac{\omega^{2}\left(\gamma-\alpha \omega^{2}\right)}{2}
$$

The Jacobian evaluated at $(r, w)$ has the form

$$
\left.\operatorname{det} \frac{\partial\left(F_{101}, F_{101}\right)}{(r, w)}\right|_{(r, w)=\left(r^{*}, w^{*}\right)}=\frac{\gamma^{2}-\alpha^{2} \omega^{4}}{2 \omega^{6}}
$$

which is nonzero by hypothesis.

From it, if $\gamma^{2}-\alpha^{2} \omega^{4}>0$ the system (6) has a unique periodic orbit bifurcating from the origin.

Note that when $\gamma=\alpha \omega^{2}$, the first-order averaging theory cannot be used. In order to apply the second-order averaging theory, we compute

$$
\left(\begin{array}{ll}
\frac{\partial F_{11}}{\partial r} & \frac{\partial F_{11}}{\partial w} \\
\frac{\partial F_{12}}{\partial r} & \frac{\partial F_{12}}{\partial w}
\end{array}\right)\left(\begin{array}{l}
\int_{0}^{\theta} F_{11}(s, r, w) d s \\
\int_{0}^{\theta} F_{12}(s, r, w) d \theta
\end{array}\right)+\left(\begin{array}{l}
F_{21}(\theta, r, w) \\
F_{22}(\theta, r, w)
\end{array}\right) .
$$

Computing the integral of this expression at the interval $[0,2 \pi]$ and dividing by $2 \pi$, we have

$$
\begin{aligned}
F_{201}(r, w)= & -r\left(r^{2} \pi-2 \beta r \omega^{2}-3 w \beta \omega-2 \omega^{2} \alpha w \pi+\alpha \omega^{5} \beta\right) / \omega^{8} . \\
F_{202}(r, w)= & -\left(7 \beta r^{2} \omega^{5}+6 \omega^{3} \beta w^{2}-4 \omega^{8} \alpha \beta r+12 w \beta r \omega^{4}\right. \\
& -8 w^{3} \pi-6 \omega^{7} \beta \alpha w-4 \omega^{8} \alpha^{2} w \pi+2 \omega^{6} \alpha r^{2} \pi \\
& \left.+12 \omega^{4} \alpha w^{2} \pi\right) / 4 \omega^{10} .
\end{aligned}
$$

Let us now consider the system

$$
\left\{\begin{array}{l}
F_{201}(r, w)=0 \\
F_{202}(r, w)=0
\end{array}\right.
$$

If we solve the first equation with respect to $w$, we obtain

$$
w=\frac{r^{2} \pi-2 \beta r \omega^{2}+\omega^{5} \alpha \beta}{\omega(2 \alpha \pi \omega+3 \beta)} .
$$

Substituting into the second equation gives 


$$
\begin{aligned}
& \left(\frac{2 \pi^{4}}{\omega^{13}(2 \alpha \pi \omega+3 \beta)^{3}}\right) r^{6}-\left(\frac{12 \pi^{3} \beta}{\omega^{11}(2 \alpha \pi \omega+3 \beta)^{3}}\right) r^{5} \\
& +\left(-3 \frac{\alpha \pi^{3}}{\omega^{8}(2 \alpha \pi \omega+3 \beta)^{2}}-3 / 2 \frac{\beta \pi^{2}}{\omega^{9}(2 \alpha \pi \omega+3 \beta)^{2}}\right. \\
& \left.+\frac{6 \alpha \pi^{3} \beta}{\omega^{8}(2 \alpha \pi \omega+3 \beta)^{3}}+\frac{24 \pi^{2} \beta^{2}}{\omega^{9}(2 \alpha \pi \omega+3 \beta)^{3}}\right) r^{4} \\
& +\left(\frac{12 \alpha \pi^{2} \beta}{\omega^{6}(2 \alpha \pi \omega+3 \beta)^{2}}-\frac{3 \beta \pi}{\omega^{7}(2 \alpha \pi \omega+3 \beta)}\right. \\
& -\frac{16 \pi \beta^{3}}{\omega^{7}(2 \alpha \pi \omega+3 \beta)^{3}}+\frac{6 \beta^{2} \pi}{\omega^{7}(2 \alpha \pi \omega+3 \beta)^{2}} \\
& \left.+-\frac{24 \alpha \pi^{2} \beta^{2}}{\omega^{6}(2 \alpha \pi \omega+3 \beta)^{3}}\right) r^{3}+\left(-\frac{\alpha \pi}{2 \omega^{4}}\right. \\
& +\frac{\alpha^{2} \pi^{2}}{\omega^{3}(2 \alpha \pi \omega+3 \beta)}-\frac{15 \beta^{2} \alpha \pi}{\omega^{4}(2 \alpha \pi \omega+3 \beta)^{2}} \\
& -\frac{7 \beta}{4 \omega^{5}}+\frac{3 \beta \alpha \pi}{2 \omega^{4}(2 \alpha \pi \omega+3 \beta)}+\frac{24 \alpha \pi \beta^{3}}{\omega^{4}(2 \alpha \pi \omega+3 \beta)^{3}} \\
& +\frac{6 \alpha^{2} \pi^{2} \beta^{2}}{\omega^{3}(2 \alpha \pi \omega+3 \beta)^{3}}+\frac{6 \beta^{2}}{\omega^{5}(2 \alpha \pi \omega+3 \beta)} \\
& \left.-\frac{6 \alpha^{2} \pi^{2} \beta}{\omega^{3}(2 \alpha \pi \omega+3 \beta)^{2}}-6 \frac{\beta^{3}}{\omega^{5}(2 \alpha \pi \omega+3 \beta)^{2}}\right) r^{2} \\
& +\left(\frac{\beta \alpha}{\omega^{2}}-\frac{12 \pi \alpha^{2} \beta^{3}}{\omega(2 \alpha \pi \omega+3 \beta)^{3}}-\frac{2 \pi \alpha^{2} \beta}{\omega(2 \alpha \pi \omega+3 \beta)}\right. \\
& +\frac{12 \pi \alpha^{2} \beta^{2}}{\omega(2 \alpha \pi \omega+3 \beta)^{2}}-\frac{6 \beta^{2} \alpha}{\omega^{2}(2 \alpha \pi \omega+3 \beta)} \\
& \left.+\frac{6 \beta^{3} \alpha}{\omega^{2}(2 \alpha \pi \omega+3 \beta)^{2}}\right) r+\frac{3 \omega \beta^{2} \alpha^{2}}{4 \alpha \pi \omega+3 \beta} \\
& -\frac{3 \omega \beta^{3} \alpha^{2}}{2(2 \alpha \pi \omega+3 \beta)^{2}}+\frac{\omega^{2} \alpha^{3} \pi \beta}{2 \alpha \pi \omega+3 \beta} \\
& -\frac{3 \omega^{2} \alpha^{3} \pi \beta^{2}}{(2 \alpha \pi \omega+3 \beta)^{2}}+\frac{2 \omega^{2} \alpha^{3} \pi \beta^{3}}{(2 \alpha \pi \omega+3 \beta)^{3}} \\
& =0 \text {. }
\end{aligned}
$$

The maximum number of positive solutions to this equation is 6 .

Let $\bar{r}$ be one of these zeros, and $(\bar{r}, \bar{w})$ be a solution of system (12). To apply Theorem 2, the condition below is needed

$$
\left.\operatorname{det} \frac{\partial\left(F_{201}, F_{202}\right)}{(r, w)}\right|_{(r, w)=(\bar{r}, \bar{w})} \neq 0 .
$$

Thus, the solutions $(\bar{r}, \bar{w})$ of the system (12) holding (13) satisfy (i) and (ii) of Theorem 2. Thus, by the second-order averaging theory (11) has at most 6 periodic orbits. From it, we state that system (6) has at most 6 periodic orbits bifurcating from the origin ending the proof. 


\section{Numerical Verification of the Analytic Results Obtained}

We consider the generalizad Genesio differential equation

$$
\dddot{x}+a \ddot{x}+b \dot{x}+c x+\sum_{k=1}^{n}(-1)^{k} x^{2 k}=0
$$

with parameters $a=\varepsilon \alpha, b=\omega+\varepsilon \beta, c=\varepsilon \gamma$. The numerical experiments have been made for the following parameters

$$
\alpha=1, \omega=1, \beta=-0.5, \gamma=1, n=5
$$

and inital conditions $x(0), \dot{x}(0)=y(0), \ddot{x}(0)=z(0)$.

In the following pictures and tables (see Tables 1-3 and Figures 1 and 2), we obtain the initial conditions and the period of four periodic solutions for different values of $\varepsilon$ with six decimal number.

Table 1. Initial conditions and periods for $\varepsilon=0.0001,0.01,0.1$ and 0.5 .

\begin{tabular}{cccccc}
\hline & $\varepsilon$ & $x(\mathbf{0})$ & $y(\mathbf{0})$ & $z(\mathbf{0})$ & $\boldsymbol{T}$ \\
\hline Orbit 1 & 0.0001 & 0.000035 & -0.000029 & -0.000024 & 6.281763 \\
Orbit 2 & 0.01 & 0.000080 & -0.00061 & -0.00006 & 6.298837 \\
Orbit 3 & 0.1 & 0.014919 & -0.017764 & -0.0116793 & 6.446567 \\
Orbit 4 & 0.5 & 0.32917 & -0.0186038 & -0.184294 & 7.293240 \\
\hline
\end{tabular}

On the other hand, we observe period doubling increasing the value of the parameter $\varepsilon$.

Table 2. Initial conditions and periods for $\varepsilon=0.735,0.745,0.758$ and 0.7605 .

\begin{tabular}{cccccc}
\hline & $\varepsilon$ & $x(\mathbf{0})$ & $y(\mathbf{0})$ & $z(\mathbf{0})$ & $\boldsymbol{T}$ \\
\hline Orbit 5 & 0.735 & -0.381394 & -0.0267376 & 0.402723 & 8.102655 \\
Orbit 6 & 0.745 & 0.882003 & -0.0312616 & -0.305098 & 16.32508 \\
Orbit 7 & 0.758 & 0.929685 & -0.0278435 & -0.268235 & 33.10797 \\
Orbit 8 & 0.7605 & 0.938029 & -0.0243008 & -0.260449 & 66.43312 \\
\hline
\end{tabular}

The existence of a period doubling cascade numerically evidences the existence of chaos, as can be seen in the pictures of Orbits 5 to 8 . We can estimate, by means of the Feigenbaum constant $F \approx 4.669 \ldots$, the value of the epsilon parameter $\varepsilon^{*}$ for which such a phenomenon can occur. The following numerical calculations are carried out in order to obtain such estimation.

Table 3. Estimation of the $\varepsilon$ parameter.

\begin{tabular}{ccccc}
\hline Bifurcation & $\boldsymbol{n}$ & $\boldsymbol{\varepsilon}_{\boldsymbol{n}}$ & $\boldsymbol{\varepsilon}_{\boldsymbol{n}}-\boldsymbol{\varepsilon}_{\boldsymbol{n}-\mathbf{1}}$ & $\boldsymbol{F}_{\boldsymbol{n}}$ \\
\hline$T \rightarrow 2 T$ & 1 & 0.745 & - & - \\
$2 T \rightarrow 4 T$ & 2 & 0.758 & 0.013 & - \\
$4 T \rightarrow 8 T$ & 3 & 0.7605 & 0.0025 & $5.2=F+0.6$ \\
\hline
\end{tabular}

$$
\varepsilon^{*} \approx \varepsilon_{1}+\left(\varepsilon_{2}-\varepsilon_{1}\right) \frac{F}{F-1}=0.7615
$$




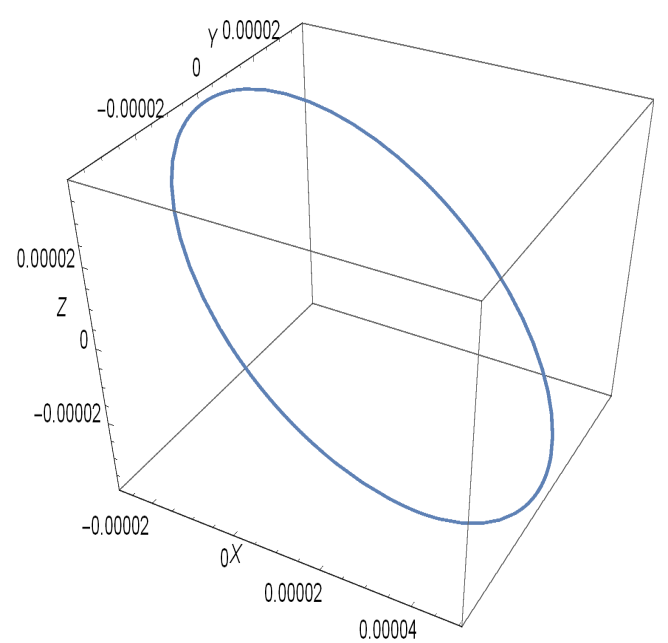

(a) Orbit 1

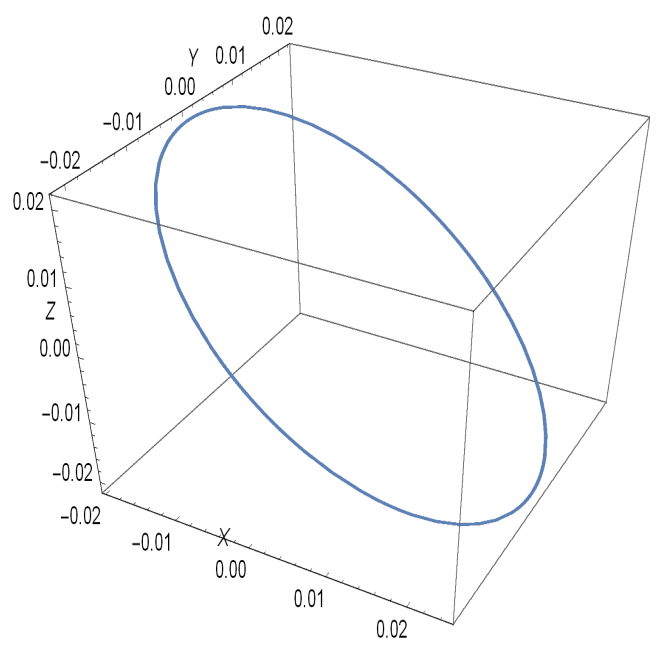

(c) Orbit 3

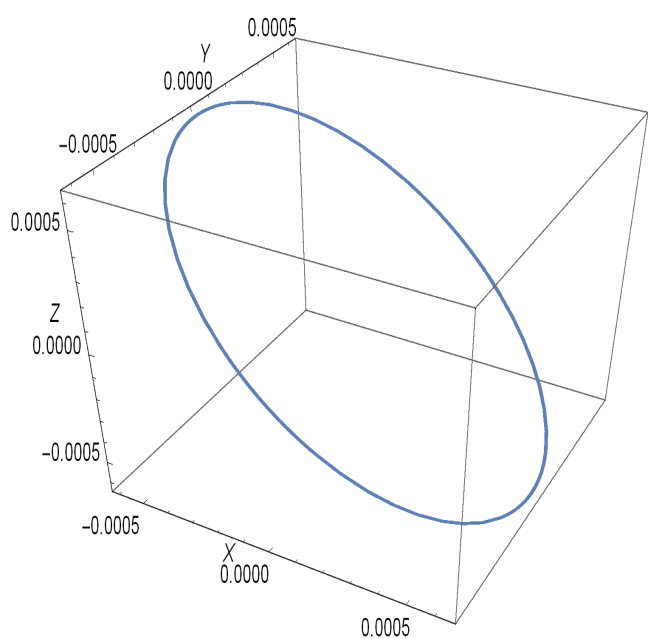

(b) Orbit 2

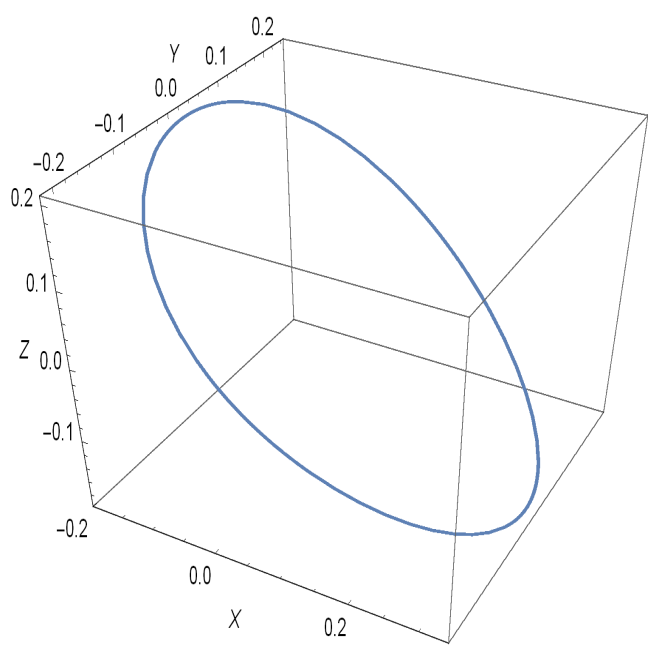

(d) Orbit 4

Figure 1. Pictures of Orbits 1 to 4 .

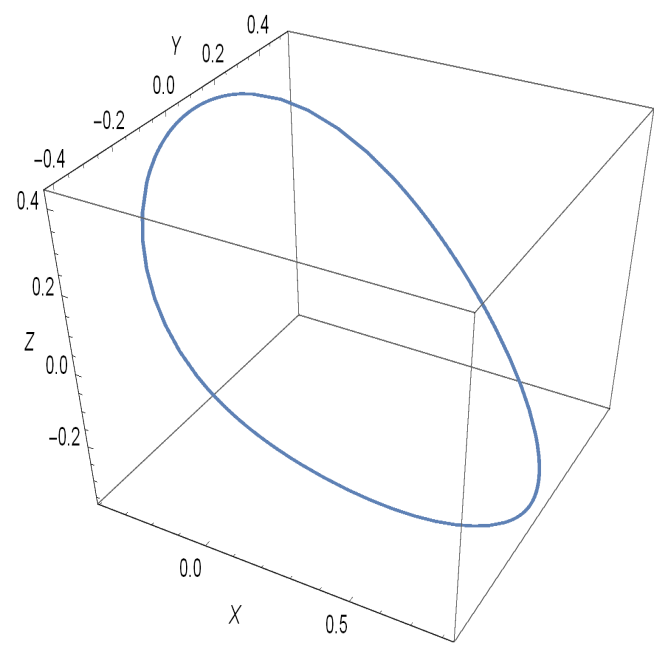

(a) Orbit 5

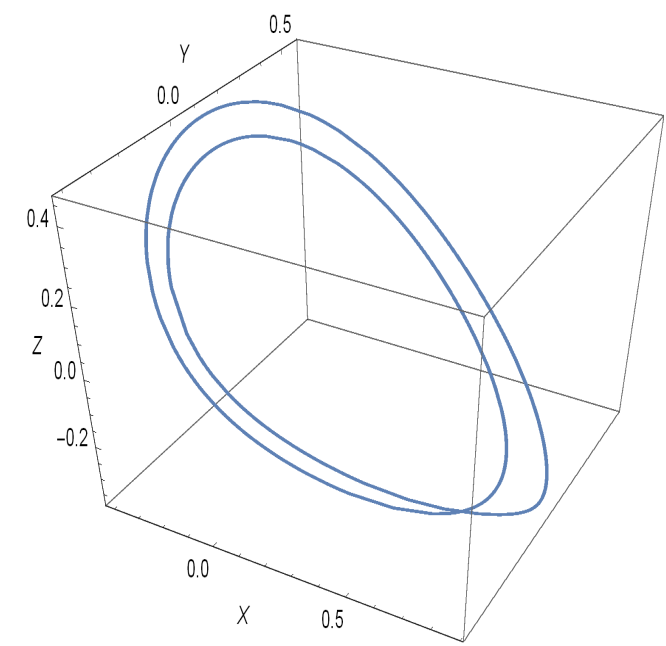

(b) Orbit 6

Figure 2. Cont. 


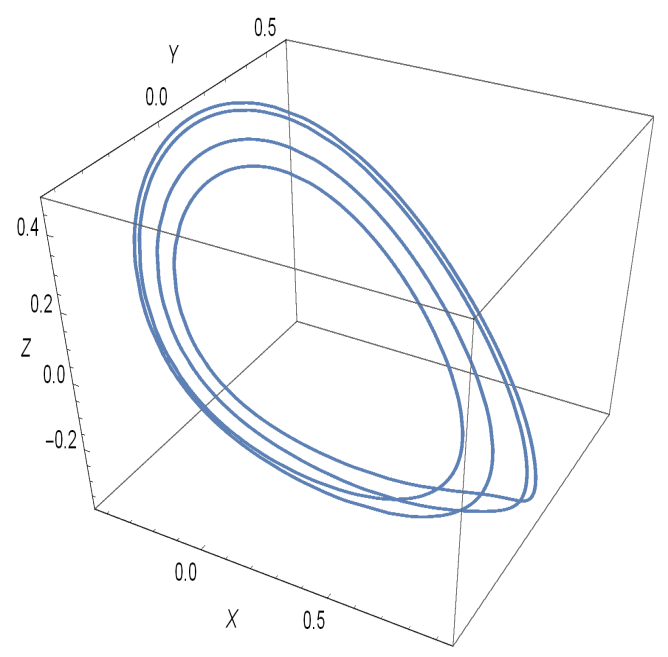

(c) Orbit 7

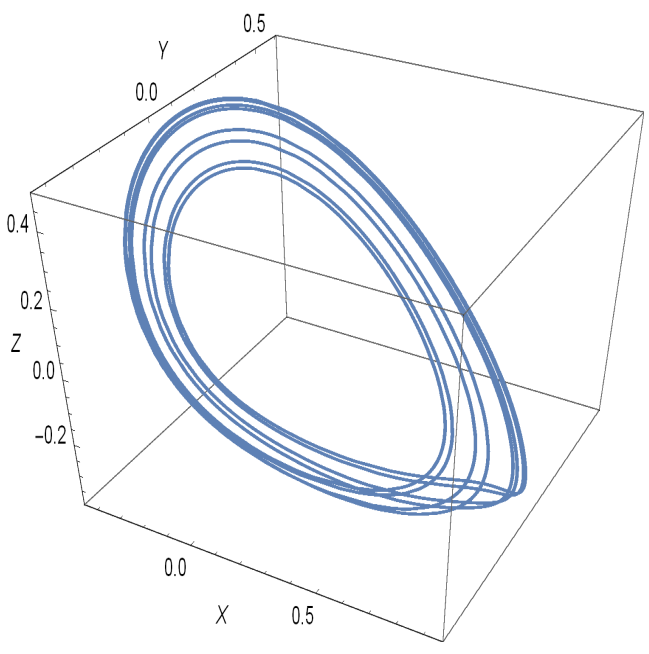

(d) Orbit 8

Figure 2. Pictures of Orbits 5 to 8.

\section{Conclusions}

The application of the averaging theory to study the existence of zero-Hopf bifurcations for a generalized Genesio differential equation gave important results about the periodic structure of these equations. Our future work will be to apply this theory to study zero-Hopf bifurcations for autonomous or nonautonomous differential equations of order $n$, with $n \geq 4$.

Author Contributions: Z.D., J.L.G.G., J.A.V. have worked in an equal way in research, develop and writing the results here presented. All authors have read and agreed to the published version of the manuscript.

Funding: This paper have been partially supported by Ministerio de Ciencia, Innovación y Universidades grant number PGC2018-097198-B-I00 and Fundación Séneca de la Región de Murcia grant number 20783/PI/18.

Conflicts of Interest: The authors declare that they have no conflict of interest.

\section{References}

1. Cardin, P.T.; Llibre, J. Transcritical and zero-Hopf bifurcations in the Genesio system. Nonlinear Dyn. 2016, 88, 547-553. [CrossRef]

2. $\quad$ Sanders, J.A.; Verhulst, F. Averaging Methods in Nonlinear Dynamical Systems; Springer: New York, NY, USA, 2007.

3. Buzzi, C.; Llibre, J.; Medrado, J. Hopf and zero-Hopf bifurcations in the Hindmarsh-Rose system. Nonlinear Dyn. 2016, 83, 1549-1556. [CrossRef]

4. Castellanos, V.; Llibre, J.; Quilantan, I. Simultaneous periodic orbits bifurcating from two zero-Hopf equilibria in a tritrophic food chain model. J. Appl. Math. Phys. 2013, 1,31-38. [CrossRef]

5. Llibre, J. Periodic orbits in the zero-hopf bifurcation of the Rössler system. Rom. Astron. J. 2014, 24, 49-60.

6. Llibre, J.; Oliveira, R.D.; Valls, C. On the integrability and the zero-Hopf bifurcation of a Chen-Wang differential system. Nonlinear Dyn. 2015, 80, 353-361. [CrossRef]

7. Llibre, J.; Mereu, A.C.; Teixeira, M.A. Limit cycles of the generalized polynomial Liénard differential equations. Math. Proc. Cambr. Philos. Soc. 2010, 148, 363-383. [CrossRef]

8. Feddaoui A., Llibre J., Chemseddine B., Makhlouf A., Periodic solutions for differential systems in $\mathbb{R}^{3}$ and $\mathbb{R}^{4}$. Appl. Math. Nonlinear Sci. 2020. [CrossRef]

9. Buică, A.; Llibre, J. Averaging methods for finding periodic orbits via Brouwer degree. Bull. Sci. Math. 2004, 128, 7-22. [CrossRef] 